\title{
Study on Performance of Laminar Taylor-Couette Flow with Different Developed Procedures
}

\author{
Xiantao Zhou, Yan Shi, Lei Jia, Yinghuang Kong \\ School of Mechanical and Power Engineering, East China University of Science and Technology, Shanghai, China \\ Email: robert_zhoucn@163.com
}

Received November 29, 2011; revised December 22, 2011; accepted December 30, 2011

\begin{abstract}
The performance of laminar Taylor-Couette flow with different developed procedures is studied by the way of computational fluid dynamics (CFD) in steady state. In order to gain a group of developed procedure in CFD, a set of convergent solutions are used as the initial value of next boundary condition, and the new set of convergent solutions are regarded as developing from the previous steady state. Three groups of developed procedures are gained from the rotating speed series of inner cylinder, respectively from the gradual increase procedure (GIP), the gradual decrease procedure (GDP) and the sudden increase procedure (SIP). It is proved that the convergent solutions of fluid control equations are different when they are solved from laminar state with the same boundary condition, the same fluid property, the same mesh grid in CFD and the same business software except that the flow states have developed from the procedures of GDP, GIP and SIP. It is shown that the developed procedure could leave behind some information in the performance of the flow. In other words, the flow between concentric rotating cylinders has somewhat memory for the procedure of its history.
\end{abstract}

Keywords: Laminar Taylor-Couette Flow; Computational Fluid Dynamics; Steady State; Memory

\section{Introduction}

Since Taylor made the initial contribution to the centrifugal instability of fluid in the fine annular gap between two concentric rotating cylinders in 1923 [1], hydro-mechanical scientists have made great achievements in the research on the performance of Taylor vortex from two ways.

One way is based on the control equations of fluid. When the small disturbances are added to the main flow between concentric rotating cylinders, the critical condition is whether the small disturbances grow with time or not. In this way, the stability condition of flow between concentric rotating cylinders is made certain [1]. Taylor deduced the first stability condition from Navier-Stokes equation and axial-symmetrical small disturbances. When inner cylinder is rotating and outer cylinder at rest, his critical Taylor number is 1708 , which is so coherent with the experiment that it is taken as one of the greatest miracles in hydro-mechanical history [2]. Subsequently, with the development of nonlinearity theory and group theory, the reasons why the Taylor vortex shows out a variety of flow patterns are made further explanations [3-5]. But so far the research on how the small disturbances impact on the solution of controlled equations is only confined to some special structures.
The other way is based on the progress of measure means and imaging technology. The researchers are endued with the capability to distinguish the various flow patterns of Taylor vortex [6]. Snyder provided empirical evidence that the nonuniqueness of wave-numbers was not an end effect but a bona fide performance of the flow [7]. In the same structure full of same fluid at the same rotating speed, Sobolik obtained the regime with 10 cells by slowly increasing the rotation rate, 12 cells by abruptly setting-up [8]. When Koschmieder studied the turbulent Taylor vortex flow, he made the rotation rate increase as slow as possible, namely at such an acceleration rate $(7 \times$ $10^{-4} \mathrm{rad} / \mathrm{s}$ ) that it took 8 hours to reach the highest studied Taylor number. Koschmieder also reported that the wavelength of laminar axisymmetric Taylor vortices were markedly different if a certain supercritical $\mathrm{T}$ was reached by a quasi-steady increase procedure or by a sudden start procedure respectively [9]. The researchers who have investigated the performance of the Taylor vortex might have realized that the prevous states could leave behind some information in the performance of flow, but unfortunately, there are few litetures on the performance of laminar Taylor-Couette flow with different developed procedures.

In order to study on the performance of laminar Tay- 
lor-Couette flow with different developed procedures, the authors use computational fluid dynamics (CFD) to obtain the characteristics of laminar Taylor-Couette flow with different developed procedures, and then discuss the performance.

\section{Numerical Method}

\subsection{Numerical Mode}

Commercial software GAMBIT2.0 is used for create mesh grid. Based on the symmetrical characteristic of the flow between concentric rotating cylinders, the geometric model is created into two-dimensional (2D): $R_{\text {in }}=100$ $\mathrm{mm}, R_{\text {out }}=110 \mathrm{~mm}, \mathrm{~L}=98.5 \mathrm{~mm}$ [9]. The gap is meshed into two grids such as L500 $\times$ R50 and L396 × R40. This two kinds of mesh grids are proved to be grid independence, so the mesh grid $396 \times 40$ is used for further analysis.

Commercial software FLUENT 6.0 version 2 ddp is used to solve the laminar flow. In this version, the solver is segregated, implicit, axisymmetric swirl, steady and absolute. The viscous model is laminar. The material of fluid has dynamic viscosity $0.0214 \mathrm{~kg} / \mathrm{m} \cdot \mathrm{s}$, density 1039 $\mathrm{kg} / \mathrm{m}^{3}$ [8]. Under-relaxation factors are 0.3 for pressure and 0.7 for momentum. The way of discretization is standard for pressure, SIMPLE for pressure-velocity coupling, first order upwind for momentum. Solution is computed from inner cylinder, and all initial values are zero unless specified. All the convergence criterions are 0.0001 unless specified. The outer cylinder and both ends are resting, and the inner cylinder is rotating with specified speed.

\subsection{Numerical Validity}

As it is well known that there are several qualitatively different steady states in the Taylor-Couette flow when the inner cylinder is rotating and the outer cylinder is at resting. The first steady state is the circular Couette flow at low Reynolds number, then following the axisymmetric Taylor-vortex flow [10]. When the Taylor number is less than the first critical Taylor number and the effect from end wall is ignored, the steady swirling velocity is known as follows:

$$
V=\frac{R_{\text {in }}^{2}}{R_{\text {in }}^{2}-R_{\text {out }}^{2}} \Omega r+\frac{R_{\text {in }}^{2} R_{\text {out }}^{2}}{R_{\text {out }}^{2}-R_{\text {in }}^{2}} \frac{\Omega}{r}
$$

where $R_{\text {in }}$ is the radius of inner cylinder, $R_{\text {out }}$ is the radius of outer cylinder, $\Omega$ is the rotational speed and $r$ is radius of annular gap between two concentric cylinders. Compared the swirling velocity from analytical solutions with that from CFD at angular speed $2.0 \mathrm{rad} / \mathrm{s}$ along the radius at the centre position of axis, we can get the Figure 1 which is shown as follows:

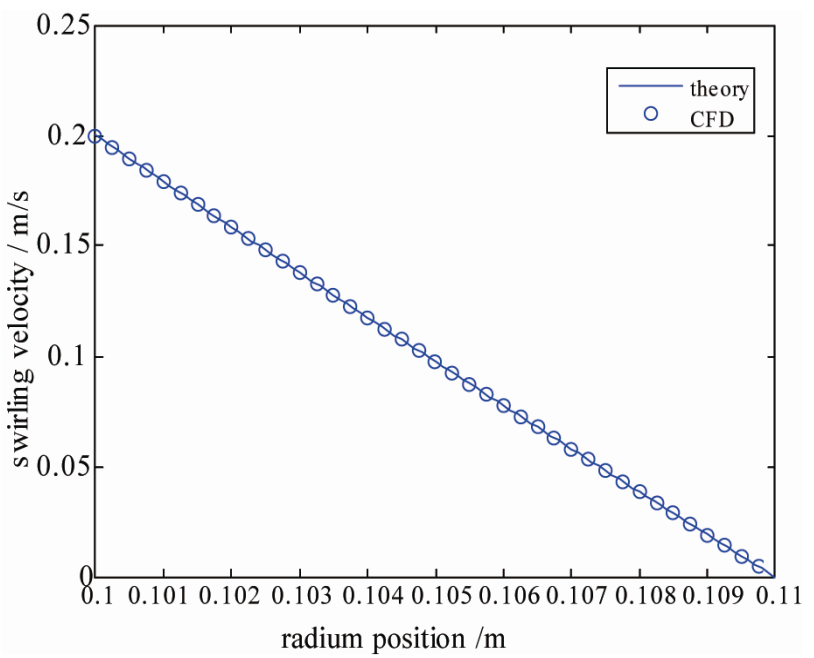

Figure 1. Compare the data from CFD with that from theory.

Figure 1 shows that the data from CFD is coherent with the data from theory. That means the way of CFD is valid for the flow between the concentric cylinders. Without introducing artificial equations to close the turbulence mode equations, using CFD to study the performances of the laminar Taylor vortex can help us to grasp the essence of Navier-Stokes equations.

\subsection{Define Developed Procedure in Simulation Process}

Although there are analytical solutions for the fluid control equations when fluid flows in the fine gap between concentric rotating cylinders [11], the ends of annular gap make the flow pattern unable to be depicted by analytical expression. Computational fluid dynamics (CFD) has given a well way to this problem especially in laminar state.

In CFD process with steady state, if a set of convergent solutions are used as the initial value of a new boundary condition, a set of new convergent solutions are taken as a new state which develops from the previous state. If the set of new convergent solutions are used as the initial value of newer boundary condition, a set of newer convergent solutions are taken as the newer state which develops from its previous newer state, and so on. Along a series of some specified boundary conditions, a group of specific developed procedure, which has evolved from a series of past states, can be gained.

There are three developed procedures to be compared with inner cylinder rotating and outer cylinder resting. The first developed procedure is that the angular velocity of inner cylinder increases from zero to $8.0 \mathrm{rad} / \mathrm{s}$ in a step of $0.2 \mathrm{rad} / \mathrm{s}$ with the convergent solution in previous step as initial values in present step, which may be named as gradual increase procedure (GIP). The second developed procedure is that the angular velocity of the inner cylin- 
der decreases from $8.0 \mathrm{rad} / \mathrm{s}$ to $0 \mathrm{rad} / \mathrm{s}$ with a step of 0.2 $\mathrm{rad} / \mathrm{s}$, which may be named as gradual decrease procedure (GDP). In order to start the second procedure, we take zero values as the initial values of $10 \mathrm{rad} / \mathrm{s}$ and then decreased to $8.0 \mathrm{rad} / \mathrm{s}$ in one step. The third procedure is that the angular velocity of the inner cylinder increases from zero to a given state suddenly and computed from the inner cylinder, which may be named as sudden increase procedure (SIP).

\section{Compare the Performance of Laminar Taylor-Couette Flow from Different Developed Procedures}

\subsection{The Radial Velocity}

Authors define a line $\mathrm{L}$ which locates at $\mathrm{r}=105 \mathrm{~mm}$ on the meridian plane of annular gap. When the angular velocity of the inner cylinder is $1.0 \mathrm{rad} / \mathrm{s}$, the radial velocities of developed procedure respectively from GDP, DIP and SIP on line L are shown in Figure 2.

Figure 2 shows that the radial velocity on the middle part of line L is nearly zero. The flow pattern usually takes as the Couette flow without the effect from end wall. In such situation, the swirl velocity can be expressed into Taylor's main flow. But there is an Ekman vortex near the static end wall. The maximum radial velocity of inward is much faster than that of outward. There is some difference among the three developed procedures at the same place, but it is not very distinct.

When angular velocity is $2.0 \mathrm{rad} / \mathrm{s}$, the radial velocities of developed procedures respectively from GDP, DIP and SIP on line L are shown in Figure 3. Figure 3 shows that there are some differences in the second vortex next to end wall.

Figure 4 is enlarged to make the difference clearly. Figure 4 shows that the second vortex near the end has

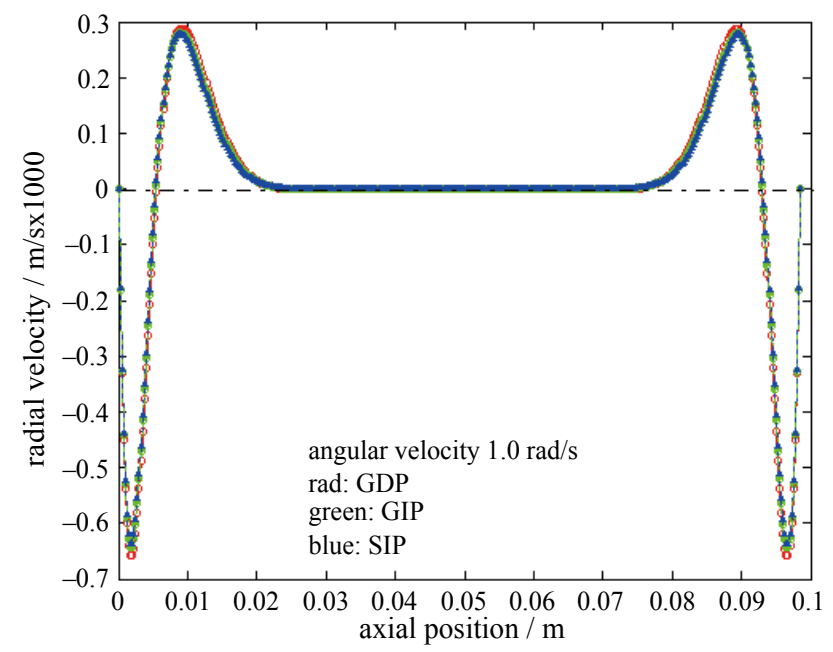

Figure 2. Distribution of the radial velocity from different procedures on line $L$ with inner cylinder rotating at $1.0 \mathrm{rad} / \mathrm{s}$.

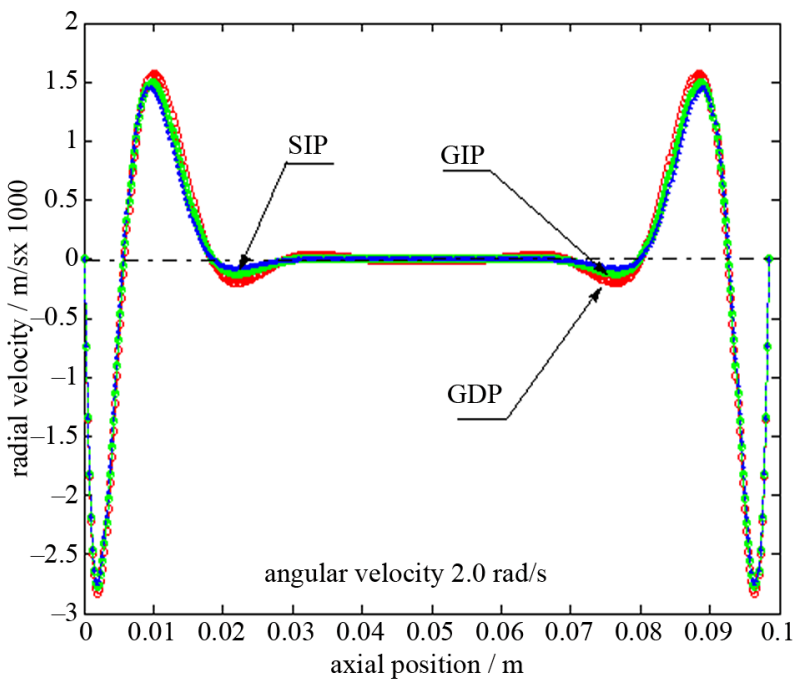

Figure 3. Distribution of the radial velocity from different procedures on line $L$ with inner cylinder rotating at $2.0 \mathrm{rad} / \mathrm{s}$.

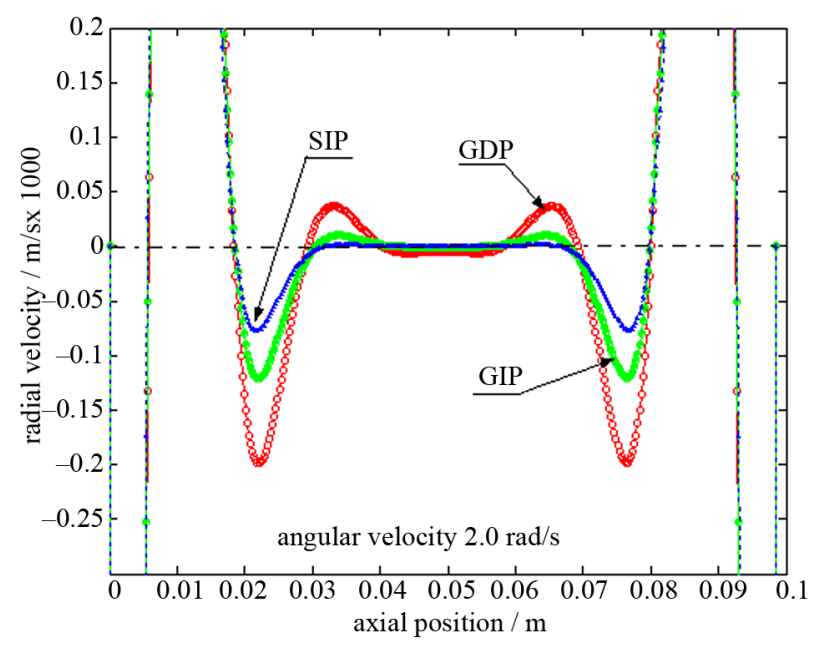

Figure 4. Partial enlarged distribution of Figure 3.

begun to develop and unroll the difference between the procedures of GDP, GIP and SIP. The inward maximum radial velocity of the second pair vortex near the end in procedure GIP is faster than that of SIP but slower than that of procedure GDP. The second pairs of vortex from GDP and GIP have developed completely, but the pair of vortex from SIP has not developed.

Figure 4 shows that the second vortex near the end has begun to develop and unroll the difference between the procedures of GDP, GIP and SIP. The inward maximum radial velocity of the second pair vortex near the end in procedure GIP is faster than that of SIP but slower than that of procedure GDP. The second pairs of vortex from GDP and GIP have developed completely, but the pair of vortex from SIP has not developed.

When angular velocity is $2.6 \mathrm{rad} / \mathrm{s}$, the radial velocities of developed procedures respectively from GDP, DIP and SIP on line L are shown in Figure 5. 


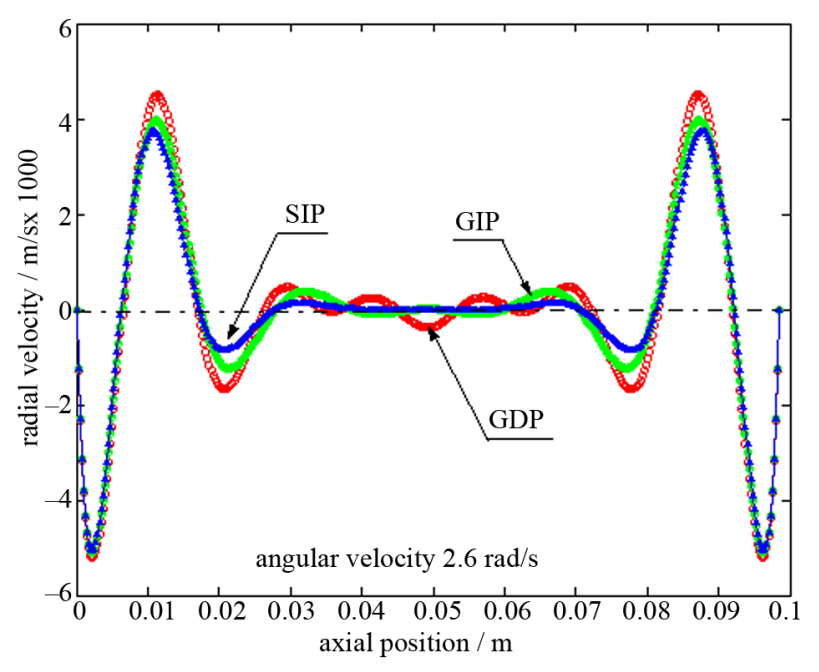

Figure 5. Distribution of the radial velocity from different procedures on line $\mathrm{L}$ with inner cylinder.

Figure 5 shows that there are 13 extreme points on line L from GDP, 8 extreme points from GIP and only 6 extreme points from SIP. The flow pattern from procedure of GDP has completely developed into the Taylor vortex full of the annular gap. The flow pattern from GIP has developed only near the ends and there is no clearvortex in the middle of line L, so does that from of SIP. At this speed, there is difference not only from the numbers of extreme points but also from the eddy structure for different developed procedures.

When angular velocity is $2.8 \mathrm{rad} / \mathrm{s}$, the radial velocities of developed procedures respectively from GDP, DIP and SIP on line L are shown in Figure 6.

Figure 6 shows that the flow patterns from the procedures of GDP and GIP have developed completely along the line $\mathrm{L}$, but the flow pattern from the procedure of

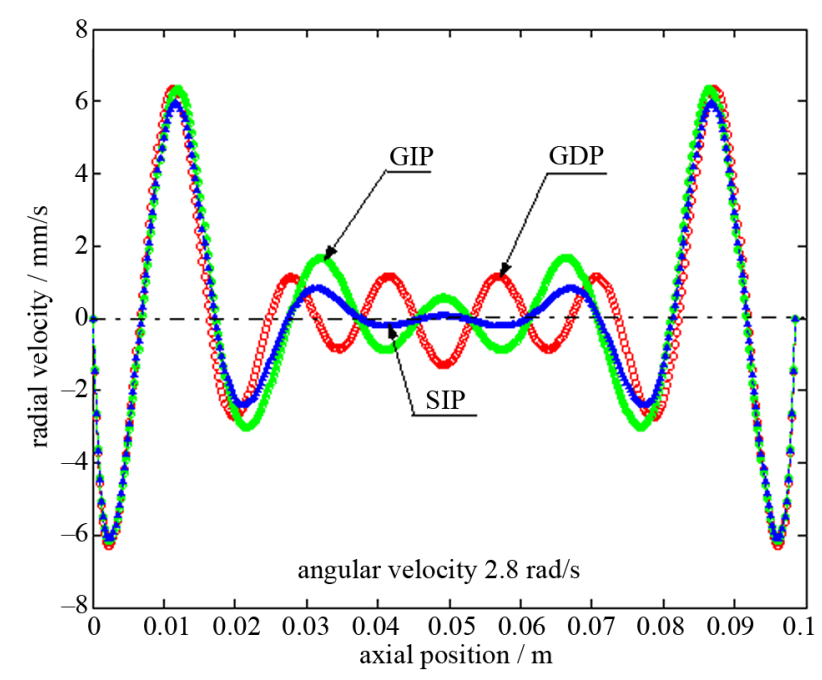

Figure 6. Distribution of the radial velocity from different procedures on line $L$ with the inner.
GDP has almost the same wave crest except for the first vortex near the end wall, which are obviously different from the wave crest from the procedure of GIP. Furthermore, the flow pattern from procedure of SIP has barely developed, especially on the middle part of line L. At this moment, the maximum radial speed is depended on the eddy near the end wall.

When angular velocity is $3.2 \mathrm{rad} / \mathrm{s}$, the radial velocities of developed procedures respectively from GDP, DIP and SIP on line L are shown in Figure 7.

Figure 7 shows that the flow patterns from the procedures of GDP, GIP and SIP have developed completely along the line $\mathrm{L}$, but the effect from the end wall is positive for the formation of eddy. The distributions of the radial velocity in the procedure of GIP and SIP are almost the same, but the number of the procedure of GDP has an eddy more than those of the procedures of GIP and SIP. The outward maximum radial velocity from the procedure GDP is less than that from procedure SIP and GIP except for the eddy near the end wall while the maximum radial velocity inwards from three procedures is almost the same. The outflows [12] in the middle from procedures of SIP and GIP are stronger than that from the procedure of GDP.

When angular velocity is $4.6 \mathrm{rad} / \mathrm{s}$, the radial velocities of developed procedures respectively from GDP, DIP and SIP on line L are shown in Figure 8.

Figure 8 shows that the flow patterns from the procedures of GDP, GIP and SIP have developed completely along the line $\mathrm{L}$. The wave crest of radial velocity in different procedures are almost the same, but the wave troughs of radial velocity in the middle of the line $\mathrm{L}$ are lower than those in the eddy near the end walls. So the effect from end wall is negative for the growth of Taylor vortex. Further, the wave troughs of the GDP are lower than

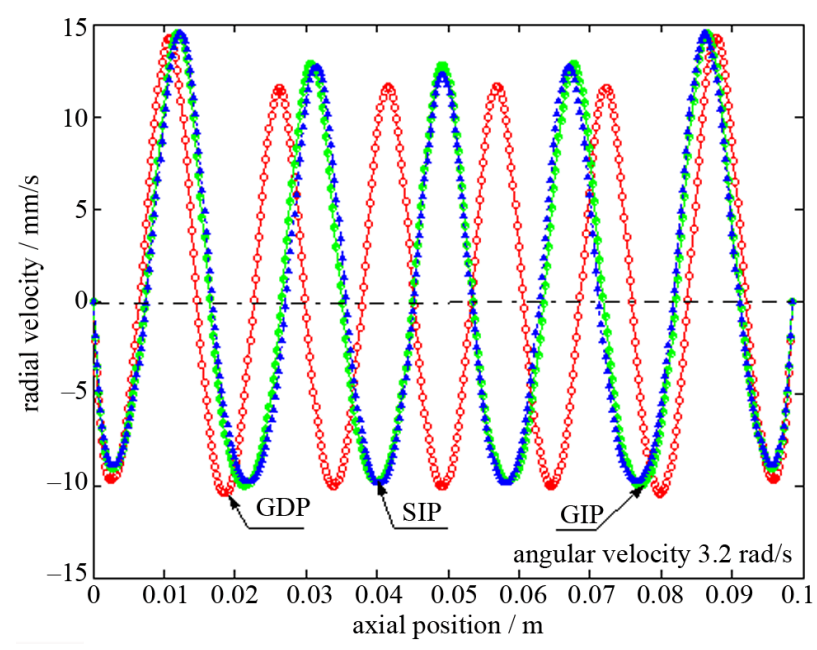

Figure 7. Distribution of the radial velocity from different procedures on line $L$ with the inner cylinder rotating at 3.2 $\mathrm{rad} / \mathrm{s}$. 


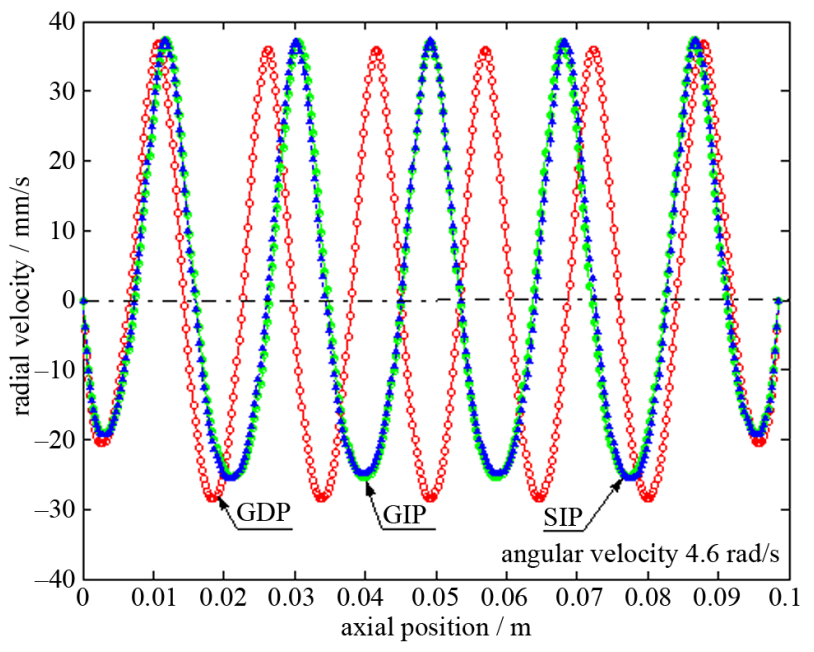

Figure 8. Distribution of the radial velocity from different procedures on line $L$ with the inner cylinder rotating at 4.6 $\mathrm{rad} / \mathrm{s}$.

those of GIP and SIP in the middle of line L, while the distribution of radial velocities on line L from the SIP and GIP are almost the same.

When angular velocity is $6.8 \mathrm{rad} / \mathrm{s}$, the radial velocities of developed procedures respectively from GDP, DIP and SIP on line L are shown in Figure 9.

Figure 9 shows that the flow patterns from the procedures of GDP, GIP and SIP have also developed completely along the line $\mathrm{L}$. The effect from the end wall is also negative for the growth of Taylor vortex, in that the wave troughs near the end walls are higher than those in the middle, but the wave crests are almost the same for different developed procedures. The wave troughs of the GDP are lower than those of GIP and SIP in the middle of line L. The inflows from GDP and SIP are stronger

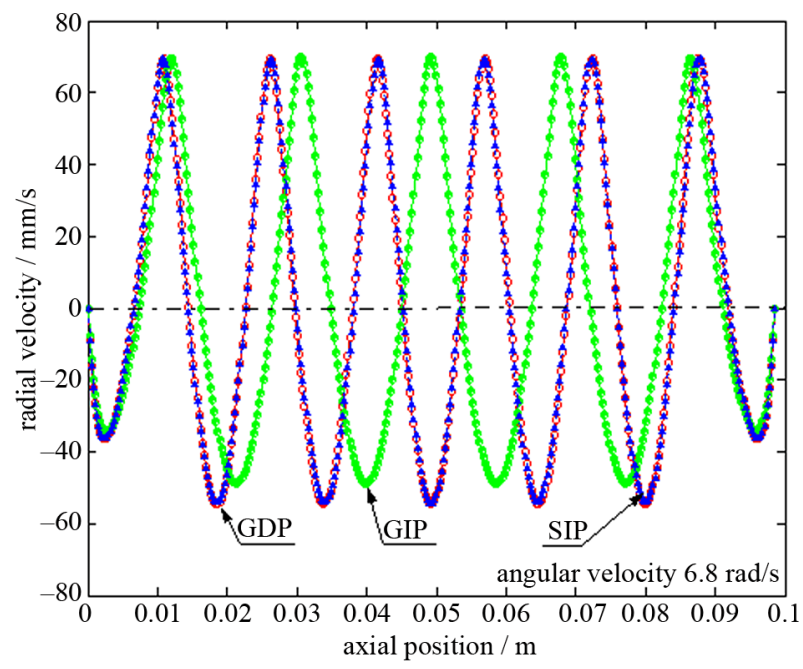

Figure 9. Distribution of the radial velocity from different procedures on line $\mathrm{L}$ with the inner cylinder rotating at 6.8 $\mathrm{rad} / \mathrm{s}$. than those from GIP in the middle of line L. The most difference is that the distribution of the radial velocity in SIP is almost the same as that in GDP in Figure 9, but that of SIP is almost the same as that in GIP at lower angular velocity such as at $4.6 \mathrm{rad} / \mathrm{s}$ or $3.2 \mathrm{rad} / \mathrm{s}$.

The distributions of radial velocity described above have shown that the laminar flows in gap between a rotating inner and stationary outer cylinder have different radial velocity characteristics and flow patterns when the flow states have developed from the procedures of GDP, GIP and SIP. It is self-evident that the Navier-Stokes equations have different radial velocity when it is solved in steady state with the same boundary condition, the same fluid property, the same mesh grid in CFD process with the same business software except that the flow has developed from the procedures of GDP, GIP and SIP.

\subsection{Compare the Maximum Axial Velocity}

Axial velocity firstly results from the Ekman layer flow when Taylor number is less than the critical Taylor number, and later from the Taylor vortex [8]. The maximum axial velocities in the GDP, GIP and SIP procedures are shown in Figure 10 in dependence on the angular velocity of the inner cylinder.

It is clear that the maximum axial velocities of three procedures are almost the same when the angular velocities are less than $2.8 \mathrm{rad} / \mathrm{s}$. Then the course bifurcates into two courses. One course is along the way of GIP, and the other is along the way of GDP. The way of SIP firstly follows the way of GIP, and then turns to the way of GDP.

It is clear that the maximum axial velocities of three procedures are almost the same when the angular velocities are less than $2.8 \mathrm{rad} / \mathrm{s}$. Then the course bifurcates into two courses. One course is along the way of GIP,

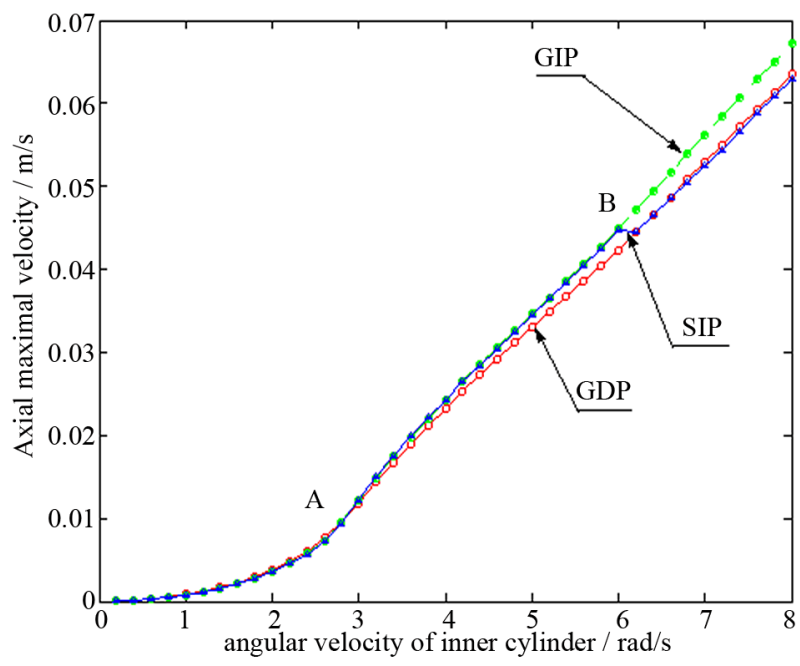

Figure 10. Comparison of the maximum axial velocities in different procedures. 
and the other is along the way of GDP. The way of SIP firstly follows the way of GIP, and then turns to the way of GDP.

If the maximum axial velocity increment in a step is divided by the step $0.2 \mathrm{rad} / \mathrm{s}$ in procedure, we can get the axial maximal velocity increasing rate per angular velocity (AMVR):

$$
\operatorname{AMVR}_{n}=\left(V_{n+1}^{a}-V_{n}^{a}\right) /\left(\Omega_{n+1}-\Omega_{n}\right)
$$

The AMVR curves of GDP, GIP and SIP are shown in Figure 11.

It is clear that each curve respectively from GDP and GIP can be divided into three parts with different trends. The first part is located before point A, where the maximal axial velocity depends only on the Ekman vortex which is caused by the end wall. The second is located from point $A$ to point $B$, where maximal axial velocity depends on the synergy between the Ekman vortex and the Taylor vortex. The third is located after point B, where maximal axial velocity depends only on the Taylor vortex. There is an interest phenomenon in Figure 10 that there is a singular point in the procedure of SIP near at $6.0 \mathrm{rad} / \mathrm{s}$, which is unlike in the procedures of GDP and GIP. The AMVR curve of GIP goes a different way from that of GDP. The AMVR curve of SIP is more uneven than that of both GDP and GIP.

The maximum axial velocities described above have also shown that the laminar Taylor-Couette flows have different maximum axial velocity when the flows have developed from the procedures of GDP, GIP and SIP. It is also proved that the Navier-Stokes equation has different maximum radial velocity when it is solved in steady state with the same boundary condition, the same fluid property, the same mesh grid in CFD process with the same business software except that the flow has developed from the procedures of GDP, GIP and SIP.

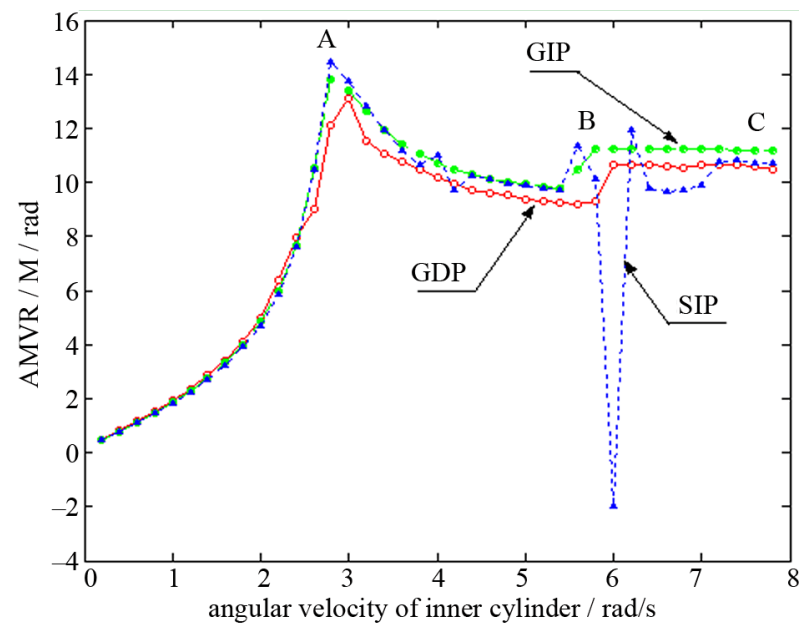

Figure 11. Comparison of the AMVR of different procedures with the angular velocity.

\subsection{Compare the Maximum Radial Velocity}

The maximum radial velocities respectively from GDP, DIP and SIP are shown in Figure 12 in dependence on the angular velocity of the inner cylinder.

It is very clear that the curve of GIP is different from that of GDP, but the curve of SIP goes to the same way with that of DIP first, then turns to the way of GDP. The trend of maximum radial velocity is similar to that of the maximum axial velocity varying with the angular velocity of the inner cylinder.

If the increment of maximum radial velocity in concentric cylinders with the inner cylinder rotating and the outer cylinder at rest is divided by the step $0.2 \mathrm{rad} / \mathrm{s}$ in procedure, we can get the radial maximal velocity increasing rate per angular velocity (RMIR):

$$
\operatorname{RMIR}_{n}=\left(V_{n+1}^{r}-V_{n}^{r}\right) /\left(\Omega_{n+1}-\Omega_{n}\right)
$$

Figure 13 shows that the RMIR of different procedures vary with the angular velocity of the inner cylinder.

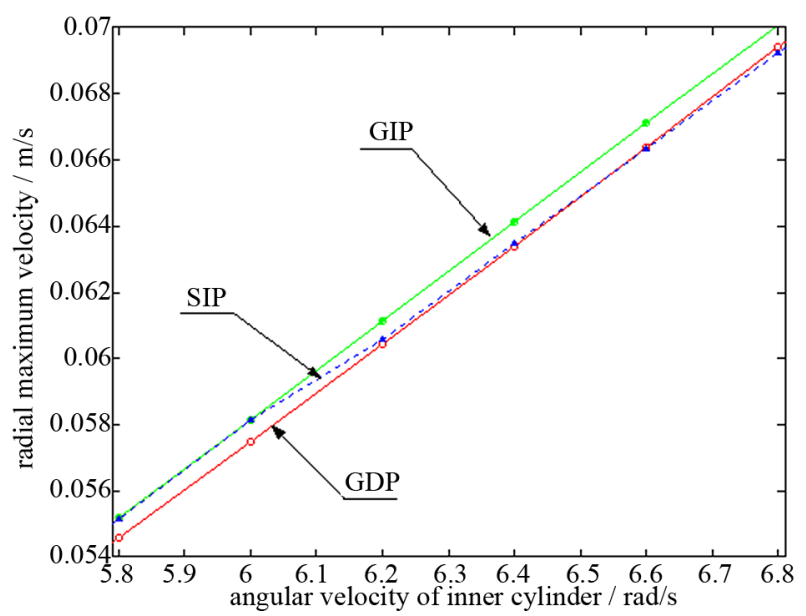

Figure 12. Comparison of the radial maximum velocities in different procedure in partial section.

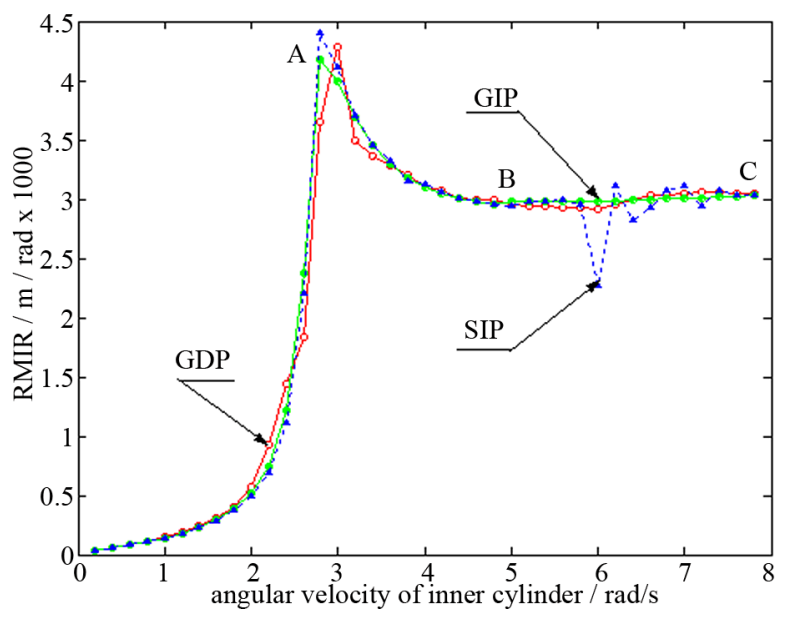

Figure 13. Comparison of the RMIR of different procedures on the angular velocity of the inner cylinder. 
The curves of the procedures could be divided into three different kinds of sections. The first section increases quickly as exponential way before point $\mathrm{A}$. The second section decreases quickly from point $\mathrm{A}$ to point $\mathrm{B}$. The third section hardly changes, despite the angular velocity increases. The course of SIP has more mutations on its way. The curve of GDP travels across the curve of GIP near at the speed $6 \mathrm{rad} / \mathrm{s}$.

According to the above analysis on the maximum radial velocity, it is self-evident that the performances of the different procedures show different characteristics at the same conditions for the same fluid in steady states near the first critical point.

The maximum radial velocities described above have shown that the laminar Taylor-Couette flows have different maximum radial velocity when the flows develop from the procedures of GDP, GIP and SIP. It shows that the Navier-Stokes equation has different maximum radial velocity when it is solved in steady state with the same boundary condition, the same fluid property, the same mesh grid in CFD process with the same business software except that the flow has developed from the procedures of GDP, GIP and SIP.

\subsection{Compare the Viscous Moment on Inner Cylinder}

The viscous moment on inner cylinder was reported by many researchers [13]. If we focus on the speed near the critical Taylor number, for example in Figure 14, we can find that the curves of the viscous moments on the inner cylinder from different procedures of GDP, GIP and SIP are departing after the critical point.

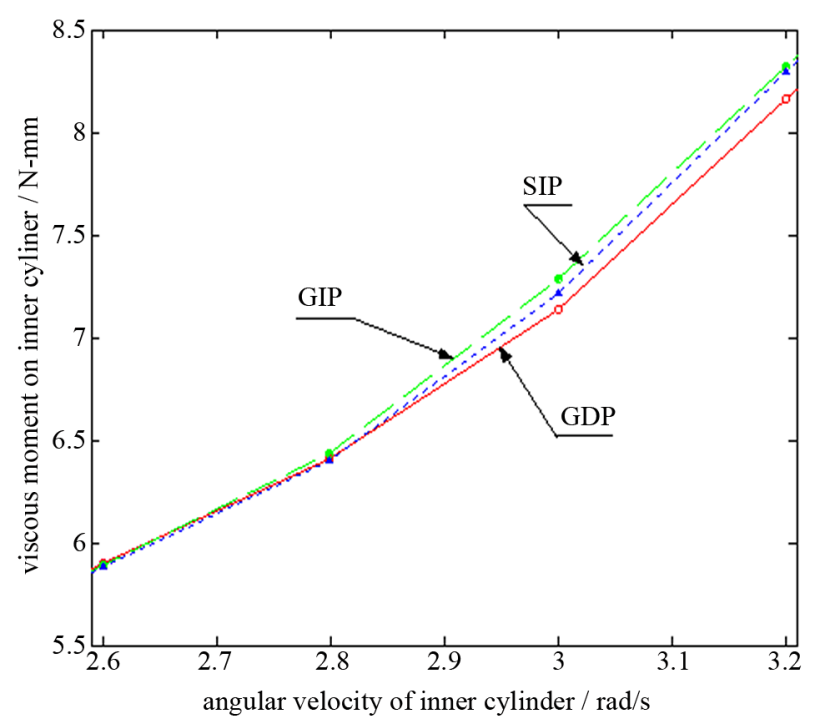

Figure 14. Viscous moments on inner cylinder from procedures of GDP, GIP and SIP as a function of angular velocity near critical Taylor number.
If the increment of viscous moment on inner cylinder in each step is divided by the step $0.2 \mathrm{rad} / \mathrm{s}$ in procedure, we can get the increment of viscous moment on inner cylinder per angular velocity (IVMPA):

$$
\operatorname{IVMPA}_{n}=\left(M_{n+1}^{\text {inner }}-M_{n}^{\text {inner }}\right) /\left(\Omega_{n+1}-\Omega_{n}\right)
$$

Figure 15 shows the variation of the IVMPA with the angular velocity of inner cylinder. When the angular velocity is less than $2.4 \mathrm{rad} / \mathrm{s}$, the IVMPA of GDP, GIP and SIP are almost the same, in that the Taylor number is less than the critical Taylor number. When the angular velocity is more than $3.0 \mathrm{rad} / \mathrm{s}$, the IVMPA curves of GDP, GIP and SIP go on various ways. The way of SIP is almost on the same way of GDP when angular velocity of the inner cylinder is between $4.0 \mathrm{rad} / \mathrm{s}$ to $5.5 \mathrm{rad} / \mathrm{s}$ firstly; and then the way of SIP turns to the way of GDP at about $6.0 \mathrm{rad} / \mathrm{s}$.

The viscous moments on inner cylinder described above have shown that the viscous moments on inner cylinder have different characteristics when the flows have developed from the procedures of GDP, GIP and SIP. It proved that the Navier-Stokes equation has different viscous moments on inner cylinder when it is solved in steady state with the same boundary condition, the same fluid property, the same mesh grid in CFD process with the same business software except that the flow has developed from the procedures of GDP, GIP and SIP.

\subsection{Compare the Viscous Moment on End Wall}

The viscous moments on end wall from GDP, GIP and SIP procedures depending on angular velocity of inner cylinder are shown in Figure 16. It shows that the way of SIP turns from the way of GIP to the way of GDP at about $6.1 \mathrm{rad} / \mathrm{s}$ as the same in Figure 15.

If the increment of viscous moment on one of the end walls in each step is divided by the step $0.2 \mathrm{rad} / \mathrm{s}$ in pro-

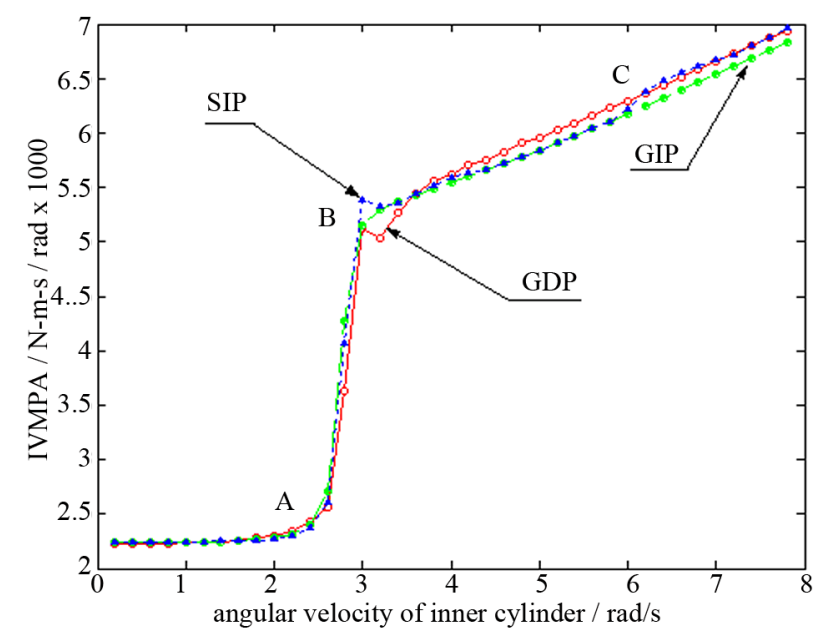

Figure 15. Variation of the IVMPA with the angular velocity of inner cylinder. 


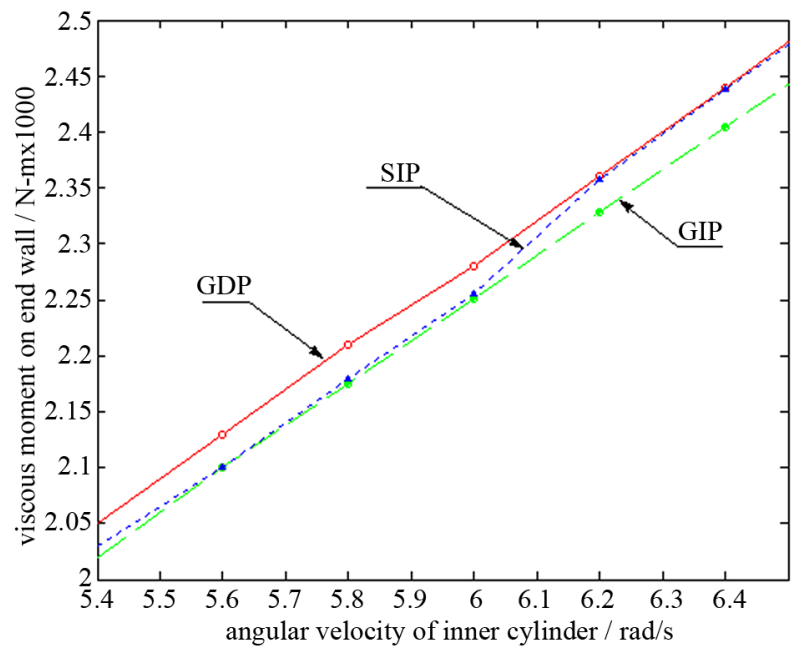

Figure 16. Viscous moments on end varying with angular velocity near the transition point.

cedure, we can get the increment of viscous moment on end wall per angular velocity (IMW):

$$
\mathrm{IMW}_{n}=\left(M_{n+1}^{\text {wall }}-M_{n}^{\text {wall }}\right) /\left(\Omega_{n+1}-\Omega_{n}\right)
$$

The curves of IMW depending on angular velocity from procedures of GDP, GIP and SIP are shown in Figure 17. In Figure 17, the IMW curves are almost the same for various procedures before the critical Taylor number. After that, the curve of GDP differs from that of GIP. The curve of SIP firstly has nearly the same way with that of GIP before $6 \mathrm{rad} / \mathrm{s}$, and then turns to the way of GDP after $6 \mathrm{rad} / \mathrm{s}$. The curves of GDP and DIP are smoother than that of SIP especially near the speed of $6 \mathrm{rad} / \mathrm{s}$.

There are some remarkable points on the way of SIP near at $6.0 \mathrm{rad} / \mathrm{s}$. The authors insert simulated values near at $6 \mathrm{rad} / \mathrm{s}$, whose convergence criterion of continuity and velocity are set to be 0.00001 which is a tenth of the previous convergence criterion 0.0001 . The IMW curves of

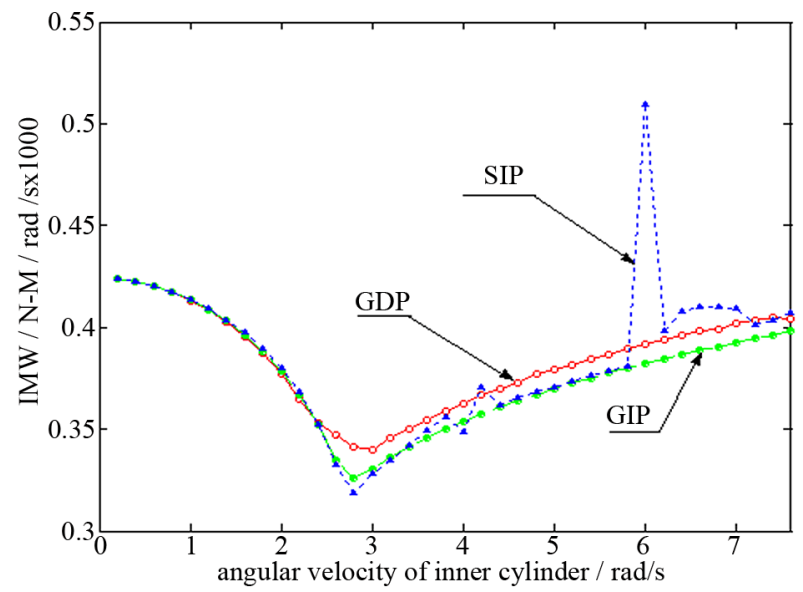

Figure 17. IMW in dependence angular velocity of inner cylinder. the GDP and GIP with convergence criterion 0.0001 and the SIP with convergence criterion 0.00001 are shows in Figure 18.

Like reported above, the IMW curve of SIP with convergence criterion 0.00001 is almost coincident with that of GIP with convergence criterion 0.0001 before $5.96 \mathrm{rad} / \mathrm{s}$, and it is also almost coincidence with that of GDP with convergence criterion 0.0001 after $6.07 \mathrm{rad} / \mathrm{s}$.

The viscous moments on end wall described above have shown that the viscous moments on end wall have different characteristics when the flows have developed from the procedures of GDP, GIP and SIP. It proved that the Navier-Stokes equation has different viscous moments on end wall when it is solved in steady state with the same boundary condition, the same fluid property, the same mesh grid in CFD process with the same business software except that the flow has developed from the procedures of GDP, GIP and SIP. It also proved that the difference is not from the convergence criterion.

\section{Discussions}

According to above contrast, there are several numerical solutions to the fluid control equations with the same boundary condition in steady laminar states from different procedures. In past, the solution of fluid control equations was usually taken for granted as the real flow so that the solution to the fluid control equations was sometimes thought of being the one and only.

In reality, it is well known that there is always perturbbation in the fluid. One kind of perturbation is from the surroundings such as the perturbation from outer forces as well as the roughness of its wall, and the other is from fluid itself such as the macro-behavior of molecular motion, in which one of the well known perturbations is the Brownian motion. When the perturbation is specified to

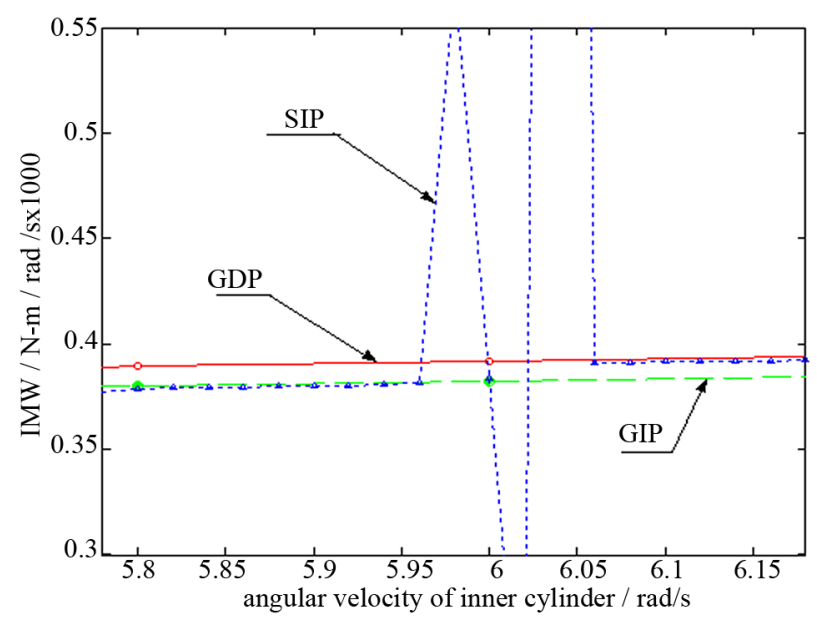

Figure 18. Comparison of the SIP points with convergence criterion 0.00001 to the different procedures with convergence criterion 0.0001 . 
be the symmetrical form, Taylor deduced the theoretical predictions in his paper, and found that there would be a definite speed at which the perturbation would suddenly make the Taylor vortex appearance. Although mathematicians could not make certain the condition so as to estimate whether the general small perturbation could grow in the flow governed by fluid control equations, the general small perturbation could come from the truncation error in CFD process, so that laminar Taylor vortex can be gained in the way of numeric solution with the small perturbation grown. So the small perturbation equations may be taken as a kind of condition beyond the stability.

As having proved above, there are different steady solutions to the fluid control equations at the same boundary condition, the same fluid property, the same mesh grid in CFD process with the same business software except that the flow has developed from different procedures of GDP, GIP and SIP. It was reported in the work of Koschmieder and Burkhalter [14] that the wave length of laminar axisymmetric Taylor vortices was significantly different if a certain supercritical Taylor number was reached either by quasi-steady increasing to the given angular velocity or by sudden start to the given angular velocity. Koschmieder also found that when a wavelength was once established by some procedure, it did not change in some rang with subsequently increased slowly or quickly. And the wavelength obtained from the sudden starts was smaller than that from the slow acceleration experiments [9]. So the developed procedure, in which the present state has undergone, should be taken as another condition of getting the real flow solution beyond the stability. In other word, the present flow state may include the information of its past, namely the flowing fluid may have the memory to some degrees.

From the view of solving fluid control equation, steady boundary condition has been generally accepted. The perturbation condition has also been accepted by researchers in hydrodynamic stability. What needs to be emphasized is that the developed procedure can leave behind some information of its history in present state so that the solutions to fluid control equations are different when the flows undergo different developed procedures. By distinguishing the details in the flow performance, we may deduce what the fluid has undergone to some degrees. In other words, the flow between concentric rotating cylinders has somewhat memory for the procedure of its history.

\section{Conclusions}

The performances of laminar Taylor-Couette flow with different developed procedures have shown that the solutions of fluid control equations are different when they are solved in steady state with the same boundary, the same fluid property, the same mesh grid in CFD process with the same business software except that the flows have developed from the procedures of GDP, GIP and SIP.

The developed procedure should be taken as the necessary condition in order to make the solution of fluid control equations become coincident with the reality beside the boundary condition and the perturbation in steady state. The developed procedure can leave behind some information of its history in the present state.

By distinguishing the details in the flow performance, we could deduce what the fluid has undergone to some degrees. In other words, the flow between concentric rotating cylinders has somewhat memory for the procedure. This may be a way of understanding the history of the present state for the fluid.

\section{Acknowledgements}

X. T. ZHOU thanks Prof. Pan Jiazhen and Prof. Chu Liangyin for their useful discussions. This work is supported by the National Natural Science Foundation of China (Grant No. 50876032).

\section{REFERENCES}

[1] G. I. Taylor, "Stability of a Viscous Liquid Contained Between Two Rotating Cylinders," Philosophical Translations of the Royal Society A, Vol. 223, No. 605-615, 1923, pp. 289-343. doi:10.1098/rsta.1923.0008

[2] P. A. Drazin and W. H. Reid, "Hydrodynamic Stabilit," Cambridge University Press, New York, 1981.

[3] M. Renardy, Y. Renardy, R. Sureshkumar and A. N. Beris, "Hopf-Hopf and Steady-Hopf Mode Interactions in Taylor-Couette Flow of an Upper Convected Maxwell Liquid," Journal Non-Newtonian Fluid Mechanics, Vol. 63, No. 1, 1996, pp. 1-31. doi:10.1016/0377-0257(95)01415-2

[4] D. G. Thomas, B. Khomami and R. Sureshkumar, "Nonlinear Dynamics of Viscoelastic Taylor-Couette Flow: Effect of Elasticity on Pattern Selection, Molecular Conformation and Drag," Journal of Fluid Mechanics, Vol. 620, 2009, pp. 353-382. doi: $10.1017 / \mathrm{S} 0022112008004710$

[5] Andrew Hill and Ian Stewart, "Hopf-Steady-State Mode Interactions with O (2) Symmetry," Dynamics and Stability of Systems, Vol. 6, No. 2, 1991, pp. 149-171. doi:10.1080/02681119108806113

[6] J. Parker and P. Merati, "Investigation of Turbulent Taylor-Couette Flow Using Laser Doppler Velocimetry in a Refractive Index Matched Facility," Journal of Fluids Engineering, Vol. 118, No. 4, 1996, pp. 810-818. doi:10.1115/1.2835513

[7] H. A. Snyder, "Change in Wave-Form and Mean Flow Associated with Wavelength Variation in Rotating Couette Flow Part 1," Journal of Fluid Mechanics, Vol. 35, No. 2, 1969, pp. 337-352. dio: $10.1017 / \mathrm{S} 0022112069001145$ 
[8] V. Sobolik, B. Izrar, F. Lusseyran and S. Skali, "Interaction between the Ekman Layer and the Couette-Taylor Instability," International Journal of Heat and Mass Transfer, Vol. 43, No. 24, 2000, pp. 4381-4393. doi:10.1016/S0017-9310(00)00067-3

[9] E. L. Koschmieder, "Turbulent Taylor Vortex Flow," Journal Fluid Mechanics, Vol. 93, No. 3, 1979, pp. 515-527. doi:10.1017/S0022112079002639

[10] C. D. Andereck, S. S. Liu and H. L. Swinney, "Flow Regimes in a Circular Couette System with Independently Rotating Cylinders," Journal Fluid Mechanics, Vol. 164, No. 3, 1986, pp. 155-183. doi:10.1017/S0022112086002513

[11] R. J. Cornish, "Flow of Water through Fine Clearance with Relative Motion of the Boundaries," Proceedings of the Royal Society A, Vol. 140, No. 840, 1933, pp. 227-240. doi:10.1098/rspa.1933.0065

[12] T. W. Steven and M. L. Richard, "Spatio-Temporal Character of Non-Wavy and Wavy Taylor-Couette Flow," Journal Fluid Mechanics, Vol. 364, 1998, pp. 59-80. doi: $10.1017 / \mathrm{S} 0022112076000098$

[13] J. A. Cole, "Taylor Vortex Instability and Annulus-Length Effects," Journal of Fluid Mechanics, Vol. 75, No. 1, 1976, pp. 1-15. doi:10.1017/S0022112076000098

[14] J. E. Burkhalter and E. L. Koschmieder, "Steady Supercritical Taylor Vortices after Sudden Starts," Physics of Fluids, Vol. 17, No. 11, 1974, pp. 1929-1935. doi:10.1063/1.1694646 